\title{
ANÁLISIS DE FORMACIÓN MUSICAL EN PROGRAMAS CURRICULARES DE EDUCACION PARVULARIA CHILENA
}

\section{ANALYSIS OF MUSICAL TRAINING IN CURRICULAR PROGRAMS OF CHILEAN PRESCHOOL EDUCATION}

\section{Emanuel Cerebello-González'; \\ Eduardo Sandoval-Obando ${ }^{2}$}

Chile

\section{RESUMEN}

Existe evidencia científica acerca del impacto positivo de la música en el desarrollo biopsicosocial del ser humano. Sin embargo, las instituciones académicas que forman a

$1 \quad$ Musicoterapeuta Facultad de Psicología, Universidad de Buenos Aires, Diplomado en Rítmica Dalcroze. Magíster en Docencia Universitaria; Director OTEC Kintsugi Spa. Académico adscrito a la Facultad de Educación, Universidad SEK (Chile). Correspondencia a: emanuel.gonzalez@usek.cl ORCID: https://orcid.org/0000-0002-2653-4583 2 Psicólogo, Magíster en Educación; Doctor en Ciencias Humanas y Postdoctorado@ en Desarrollo Evolutivo. Académico perteneciente a la Escuela de Psicología, Grupo de "Investigación en Educación, Cultura y Transformación Social" (ICET) e Investigador asociado al Instituto de Estudios Sociales y Humanísticos, Facultad Ciencias Sociales y Humanidades. Universidad Autónoma de Chile (Temuco - Chile). ORCID: https://orcid.org/0000-0001-7471$\underline{6536}$ educadoras de párvulo le asignan escasa importancia a la formación de competencias musicales para el desempeño profesional. De hecho, las bases curriculares de educación parvularia recientemente publicadas por el Ministerio de Educación en Chile no dan cuenta sobre la importancia de los recursos musicales como un lenguaje artístico autónomo y de utilización transversal en los tres ámbitos de experiencias para el aprendizaje que ahí se describen. El presente artículo busca exponer la relevancia curricular de la educación musical, en las instituciones que forman educadoras de párvulo en Chile. Específicamente, se realiza una evaluación crítica en torno a las bases curriculares para la educación 
parvularia adoptando un diseño cuantitativo, descriptivo, transversal y no experimental. Para la obtención de datos se analizó la carga horaria de las materias relacionadas con el lenguaje artístico, didácticas y particularmente del lenguaje musical de 23 planes de estudios de instituciones universitarias chilenas. Como criterios de inclusión y exclusión, se consideraron formaciones con mallas curriculares publicadas en sus portales web a la fecha de la investigación. Los resultados develan un bajo porcentaje de horas designadas a la expresión musical $(91 \%$ de las instituciones analizadas presenta sólo un semestre de formación en la temática), como también la ausencia de la materia en un $17 \%$ de las instituciones que participaron del estudio.

Palabras claves: Formación musical, mallas curriculares, educación parvularia, educación inicial, neurociencias, música.

\section{ABSTRACT}

There is scientific evidence about the positive impact of music on the biopsychosocial development of the human being. However, the academic institutions that form preschool educators assign little importance to the formation of musical skills for professional performance. In fact, the curricular bases of pre-school education recently published by the Ministry of Education in Chile do not give an account of the importance of musical resources as an autonomous and crosssectional artistic language in the three areas of learning experiences described there. This article seeks to expose the curricular relevance of music education in the institutions that form preschool educators in Chile. Specifically, a critical evaluation is carried out around the curricular bases for preschool education adopting a quantitative, descriptive, transversal and nonexperimental design. In order to obtain data, the hourly load of the subjects related to artistic, didactic and particularly musical language of 23 curricula of Chilean university institutions was analyzed. As inclusion and exclusion criteria, training with curricular meshes published in their web portals at the date of the investigation were considered. The results reveal a low percentage of hours designated for musical expression (91\% of the institutions analyzed have only one semester of training in the subject), as well as the absence of the subject in $17 \%$ of the institutions that participated in the study.

Keywords: Musical training, curricular meshes, preschool education, initial education, neurosciences, music.

\section{SUMÁRIO}

Existem evidências científicas sobre o impacto positivo da música no desenvolvimento biopsicossocial do ser humano. No entanto, as instituições acadêmicas que formam um educador de infância atribuem pouca importância à formação de habilidades musicais para o desempenho profissional. De fato, as bases curriculares da educação pré-escolar recentemente publicadas pelo Ministério da Educação no Chile não dão conta da importância dos recursos musicais como uma linguagem artística autônoma e transversal nos três dispositivos de experiência de aprendizagem aqui descritos. . Este artigo procura expor a relevância curricular da educação musical nas instituições que formam educadoras de infância no Chile. Especificamente, é realizada uma avaliação crítica em torno das bases curriculares da educação pré-escolar, adotando um desenho quantitativo, descritivo, transversal e não experimental. Para obter os dados, analisouse a carga horária das disciplinas relacionadas à linguagem artística, didática e específica da linguagem musical de 23 currículos de instituições universitárias chilenas. Como critério de inclusão e exclusão, considere o treinamento com malhas curriculares publicadas em seus portais na web na data da investigação. Os resultados revelam uma baixa porcentagem de horas designadas para expressão musical $(91 \%$ das instituições analisadas possuem apenas um 
semestre de treinamento na disciplina), além da ausência da disciplina em 17\% das instituições que participaram do estudo.

Palavras-chave: Treinamento musical, malhas curriculares, educação pré-escolar, educação inicial, neurociências, música.

\section{INTRODUCCIÓN}

Con base en la experiencia acumulada a lo largo de los años, los autores develan que existen escasas competencias musicales y conocimiento de los beneficios de la música en educadoras de párvulos, que les permitan desarrollar procesos de iniciación musical y desarrollo de aprendizajes significativos en sus educandos. Al parecer en Chile las prácticas pedagógicas al nivel preescolar ha mostrado importantes déficit (Guerra Zamora et al., 2017), donde la educación artística, y en particular la educación musical suele ser el campo del conocimiento más relegado del currículo, ubicándose históricamente en la periferia curricular (Arredondo, 2020). Es probable que el sistema económico no vea la conveniencia de desarrollar habilidades musicales en los sujetos sociales, ya que las pretensiones neoliberales no se interesan en las aportaciones afectivas y de educación emocional que ofrece la educación musical, (Leonard, Hafford-Letchfield, y Couchman, 2018).

Las competencias profesionales que debe poseer una educadora de párvulos son tan amplias como exigentes, y manejar estrategias pedagógicas musicales es uno de los estándares que debe poseer (Elige Educar, 2019). Sumado a ello las escuelas chilenas reconocen que no cuentan con aulas de música y la representación social que los estudiantes de escuelas públicas chilenas tienen acerca de la implementación escolar para la educación musical, es que el sistema educativo posterga la formación integral a tal punto, que vulnera los principios fundamentales de equidad y calidad en la educación musical (Angel-Alvarado y LiraCerda, 2017).

Actualmente, existen estudios científicos desde las neurociencias que constatan la influencia del ejercicio musical tanto en tareas de escucha como de producción sonora, evidenciando el impacto positivo en las funciones ejecutivas, cognitivas, mnémicas y emocionales, entre otras del individuo (Patel, 2003; Peretz y Coltheart, 2003; Bangerter y Heath, 2004; Zatorre, Chen y Penhune, 2007; Cánovas, Estévez y SánchezSanted, 2008; Forgeard et al, 2008; Albornoz, 2009; Schlaug et al, 2009; Milovanov, 2009, 2010 y 2011; Dege et al., 2011; Soria-Urios, Duque y Garcia-Moreno, 2011; Meher et al., 2013; Collins, 2013; Roden et al. 2013 y 2014; Zuk et al. 2015; Fernández y García, 2015; Custodio y Cano-Campos, 2017; Benítez, Díaz y Justel, 2017; Jaschke, Honing. y Scherder, 2018; Orozco y Gil, 2018, Román-Caballero y Lupiañez, 2019).

Por su parte, Collins (2013) ha revelado importantes diferencias en las estructuras cerebrales de personas con estudio musical y personas sin estudio musical, donde la práctica musical permitiría el desarrollo de cerebros más grandes y con un mejor funcionamiento y desempeño global, lo que invita a redefinir las políticas educativas, las estrategias pedagógicas y la relevancia de la educación musical en la jerarquía curricular.

La evidencia acumulada desde las neurociencias reiteran la necesidad de fortalecer el currículo musical frente a los legisladores y políticas públicas en general (Peñalba, 2017), para asegurar la igualdad de oportunidades de todos los niños y niñas, a través de programas educacionales robustos que permitan entrenamiento musical adecuado para obtener los beneficios múltiples en el cerebro humano que genera el quehacer musical (Barraza, 2018). 


\section{DESARROLLO}

\section{Políticas Educativas en Primera Infancia: El Caso de Chile}

La situación curricular de la educación musical se encuentra invisibilizada, siendo foco de preocupación en varios países de Iberoamérica (Aróstegui, 2016). En España, la Ley Orgánica De Educación promulgada en 2006, más conocida como LOE, ha dificultado el desarrollo de una educación musical de calidad, generando un déficit en la formación musical de los futuros maestros (Aróstegui, 2014). En Chile, se fusionaron las asignaturas de artes plásticas, educación musical, y tecnología, por lo cual cada directivo de los establecimientos educativos públicos debe decidir a cuál asignatura le entrega mayor relevancia, extendiendo la posibilidad real de reducción de las horas de música. Actualmente, el currículum vigente asigna 76 horas de música anuales de un total de 1.444 horas anuales, para los planes de estudio de $1^{\circ}$ a $4^{\circ}$ año básico. Esto es un $5.2 \%$ del total de horas, en comparación a la asignatura de lenguaje y comunicación que posee un $21 \%$ de la carga horaria, por dar un ejemplo. Este contexto se vuelve más crítico en $5^{\circ}$ y $6^{\circ}$ año de enseñanza básica, donde las horas de formación musical bajan a 38 horas anuales, un $2.6 \%$ del total de horas lectivas (Mineduc, 2013). Sumado a ello, y a pesar de estar sobre la media en Latinoamérica, las escuelas chilenas reconocen que no cuentan con aulas de música, encontrándose que una de cada cinco tendrían un espacio para su realización (Duarte, Gargiulo y Moreno, 2011). Por otra parte, la representación social que los estudiantes de escuelas públicas chilenas tienen acerca de la implementación escolar para la educación musical no es mucho más alentador, su percepción es que el sistema educativo posterga la formación integral a tal punto, que vulnera los principios fundamentales de equidad y calidad en la educación musical (Alvarado y Lira-Cerda, 2017).
En la formación inicial de educadoras de párvulos persisten críticas acerca de la actualización curricular de acuerdo a los debates internacionales (Elige Educar, 2019), y resulta clarificador explicitar que son éstas profesionales las encargadas de planificar estrategias pedagógicas musicales para el desarrollo de los infantes. Cabe entonces preguntarse si están preparadas las educadoras para desarrollar estrategias pedagógicas musicales, con toda la complejidad que el multiverso musical requiere para su apropiación, y al menos asegurar una mejora en la calidad y la transferencia de objetivos de aprendizaje en los educandos.

Bases curriculares para la Educación Parvularia 2018

En mayo del 2018, se han publicado las Nuevas Bases Curriculares para la Educación Parvularia (MINEDUC, 2018). Estas orientaciones complementan a otros tres instrumentos: Estándares de Desempeño para la Formación Docente, Estándares Orientadores para Carrera de Educación Parvularia y Marco para la Buena Enseñanza, que en su conjunción sientan las bases para entender los aprendizajes esperados en el aula, regular y promover la calidad de la educación parvularia, y las políticas chilenas en torno a ésta área (Elige Educar, 2019)

La nuevas BCEP sientan su composición estructural en la división de tres ámbitos de experiencias (desarrollo personal y social, comunicación integral, interacción y comprensión del entorno) donde se organizan y distribuyen los objetivos de aprendizaje. Una de las particularidades es que solo un ámbito (desarrollo personal y social) se vuelve transversal, vale decir, que sus objetivos de aprendizaje se deben trabajar permanentemente en los dos otros ámbitos, y no todos en combinación. Asimismo, cada ámbito tiene núcleos de aprendizaje que corresponden a focos de experiencias en torno 
a los cuales se integran y articulan los objetivos de aprendizaje. Al respecto, se describen los objetivos de aprendizaje que cada párvulo se espera consiga de acuerdo con cada nivel.

\section{Impacto de las BCEP 2018: Una Revisión Crítica}

Siendo el 2018 un año de transición, el 2019 es el primer año de implementación efectiva de las BCEP, por lo tanto aún no existen informes técnicos que evalúen su implementación (Elige Educar, 2019), en consecuencia se realizará un análisis crítico en torno a este instrumento.

En primer lugar, no se entiende que el ámbito de desarrollo personal y social sea el único que deba trabajarse de forma transversal, es decir de forma permanente. En el documento oficial se declara:

"En estas Bases Curriculares, el Ámbito de Desarrollo Personal y Social adquiere un carácter transversal en la estructura e implementación curricular, debido a la relevancia formativa que tienen sus componentes para el desarrollo humano y por lo cual se requiere que sean visibilizados de manera permanente en todas las acciones educativas que se propician con los niños y las niñas" (MINEDUC, 2018, p.38).

Esta indicación coarta, o al menos confunde, las posibilidades de diseño de experiencias de aprendizaje que puedan incluir, por qué no, los tres ámbitos de experiencias, por ejemplo: planificar una actividad de escucha y posterior improvisación de paisajes sonoros, explorando nuestro entorno natural, para desarrollar también la identidad y autonomía de nuestra comunidad. En esta simple propuesta, estamos combinando los tres ámbitos de forma transversal, utilizando un núcleo de cada ámbito, y sin jerarquía de una experiencia sobre otra.

Por otra parte, en las bases se fusiona el quehacer musical con la plástica, el teatro, la danza, la escultura, la pintura, entre otros, como si fuese todas las áreas una misma disciplina. Contextos como éste se dan a nivel global (Aróstegui, 2016), y urge la necesidad de repensar la formación inicial docente, dotándolos de herramientas musicales integrales basadas en las artes (Estévez y Rojas, 2017). Otro aspecto de tensión y discusión son los referentes teóricos que se utilizaron para la confección de las nuevas bases curriculares para educación parvularia 2018. Al revisar la bibliografía sólo se encontró un documento sobre educación musical publicado en el año 1990, lo que devela un alto grado de superficialidad y desconocimiento acerca de los hallazgos de los últimos 30 años en neurociencias y la importancia que tiene el vínculo musical temprano para el desarrollo del ser humano.

\section{MARCO METODOLÓGICO}

\section{Paradigma y Diseño del Estudio}

Desde una perspectiva cuantitativa (Kerlinger, 2002; Popper, 2008), se adopta un diseño de tipo descriptivo, no experimental, transversal, ya que se observan y evalúan fenómenos, se establecen ideas como consecuencia a lo observado, se demuestra el grado de fundamento de las ideas (Piscoya, 2009), se revisan tales ideas de acuerdo a los resultados analizados y por último se proponen nuevas observaciones para esclarecer las suposiciones o complementarlas (Hernández, Fernández y Baptista, 2016). El estudio involucra además la revisión documental, ya que se analizaron múltiples orientaciones técnicas y pedagógicas, además de las diversas mallas y planes de formación académica que poseen las educadoras de párvulos en Chile. 


\section{Caracterización de la Revisión Documental}

En este trabajo, se analizaron las mallas curriculares de las carreras de Educación Parvularia de 23 universidades y centros de formación de Chile. Así, se revisaron los planes de estudio para observar si las instituciones incluyen o no la asignatura Expresión Musical en sus mallas de Educación Parvularia, si ésta se encuentra en combinación con otras artes y la cantidad de semestres que posee la asignatura en su formación, entendiendo que cada semestre entrega una cantidad de créditos regulados por el sistema de créditos transferibles (Consejo de Rectores, 2019). Los créditos académicos SCT representan la carga de trabajo que demandará una actividad curricular al estudiante para el logro de los resultados de aprendizaje. Desde el punto de vista cuantitativo, un crédito equivale a la proporción respecto de la carga total de trabajo necesaria para completar un año de estudios a tiempo completo. La muestra fue tomada durante el mes de noviembre del 2018.

\section{Criterios de inclusión y exclusión empleados en la Revisión Documental}

Como criterio de inclusión, se consideraron de forma aleatoria las mallas curriculares de 23 universidades y/o centros de formación que tuviesen publicados sus programas, mallas curriculares o planes de estudio de Educación Parvularia en sus portales web. Las universidades que cumplieron con ese requisito fueron: Universidad Andrés Bello (UNAB), Universidad Adventista de Chile (UNACH), Universidad Católica de Valparaíso (PUCV), Universidad Católica Raúl Silva
Henríquez (UCSH), Universidad Bernardo O'Higgins (UBO), Instituto Profesional de Chile (IP), Universidad Metropolitana Ciencias de la Educación (UMCE), Universidad de Las Américas (UDLA), Universidad Pedro de Valdivia (UPV), Universidad Alberto Hurtado (UAH), Universidad Católica (UC), Universidad de Concepción (UDEC), Universidad Diego Portales (UDP), Universidad Finis Terrae (UFT), Universidad de Los Lagos (ULL), Universidad de Chile (UCH), Universidad Mayor (UMAY), Universidad Arturo Prat (UNAP), Universidad San Sebastián (USS), Universidad de Tarapacá (UTA), Universidad Autónoma (UA), Universidad SEK (USEK),Universidad Católica del Maule (UCM). Se excluyeron de la muestra las instituciones que no respondieron a los correos solicitando dicho programa, las que no poseían página web, o no contenían la publicación del programa de estudio en sus portales.

\section{Hipótesis}

El $80 \%$ de las instituciones que imparten la formación de educación parvularia en Chile incluyen al menos un semestre de la asignatura expresión musical, sin combinación con otras disciplinas en sus planes de estudio.

\section{Variables Analizadas}

Para efectos prácticos, se describen operacionalmente las variables independientes que orientaron el análisis documental (ver Tabla 1): 
Tabla 1.

Descripción Operacional de variables analizadas.

\begin{tabular}{|l|l|}
\hline Variables Analizadas & Indicador \\
\hline $\begin{array}{l}\text { La asignatura de expresión musical en carreras } \\
\text { de párvulos de universidades chilenas. }\end{array}$ & $\begin{array}{l}\text { Número de universidades que contiene la } \\
\text { asignatura recursos musicales. }\end{array}$ \\
\hline $\begin{array}{l}\text { La asignatura expresión musical en combinación } \\
\text { con plástica. }\end{array}$ & $\begin{array}{l}\text { aúmero de universidades que contiene la } \\
\text { cignatura recursos musicales en combinación }\end{array}$ \\
\hline $\begin{array}{l}\text { La asignatura expresión musical en combinación } \\
\text { con teatro. }\end{array}$ & $\begin{array}{l}\text { Número de universidades que contiene la } \\
\text { asignatura recursos musicales en combinación }\end{array}$ \\
\hline $\begin{array}{l}\text { La asignatura expresión musical en combinación } \\
\text { con danza. }\end{array}$ & $\begin{array}{l}\text { Número de universidades que contiene la } \\
\text { asignatura recursos musicales en combinación }\end{array}$ \\
\hline $\begin{array}{l}\text { La duración de la asignatura de expresión musical } \\
\text { en las carreras de educación parvularia. }\end{array}$ & $\begin{array}{l}\text { Número de semestres que contienela asignatura } \\
\text { recursos musicales en las mallas curriculares. }\end{array}$ \\
\hline
\end{tabular}

Fuente de Elaboración Propia

\section{Procedimiento de recolección de datos}

En primera instancia, se enviaron correos electrónicos a los equipos directivos de las carreras de Educación Parvularia que se encontraban ofertadas en la página del Ministerio de Educación y sitios web institucionales. Se recibieron sólo 6 respuestas y sólo una adjuntó su malla curricular, las otras cuatro solicitaron remitirse a la información publicada en sus sitios web. Ante esta situación, se resolvió realizar el análisis de acuerdo con las mallas curriculares que fueron encontradas en las páginas web de cada institución a noviembre del año 2018.

Por otro lado, no existe certeza de la cantidad de horas reales de formación en cada semestre, información sensible que es reservada por la mayoría de las casas de estudio. Esta situación puede conducir a un sesgo en la investigación. Como medida complementaria se realizó una búsqueda de egresadas de Educación Parvularia de 5 promociones de las instituciones analizadas y se les preguntó sobre la carga horaria. En los 5 casos, la respuesta fue de 3 a 4 horas semanales, lo que corresponden a una asignatura semestral de entre 60 a 78 horas. Semestrales equivalentes a 2 créditos regulados por la SCT.

\section{Delimitación de la Muestra}

A continuación, se exponen (ver la tabla 2) los datos obtenidos en la revisión documental, para delimitar la muestra seleccionada en este estudio. 
Tabla 2.

Inclusión de asignatura Expresión musical y Número de semestres.

\begin{tabular}{|c|c|c|c|c|c|c|c|c|}
\hline \multicolumn{4}{|c|}{ Tabla de inclusión de asignatura } & \multicolumn{5}{|c|}{ Tabla con número de semestre } \\
\hline $\begin{array}{l}\text { Universidad/ } \\
\text { Asignatura }\end{array}$ & $\begin{array}{l}\text { Expresión } \\
\text { Musical }\end{array}$ & $\begin{array}{l}\text { Música / } \\
\text { Plástica }\end{array}$ & $\begin{array}{l}\text { Música/ } \\
\text { Teatro }\end{array}$ & $\begin{array}{l}\text { Música/ } \\
\text { Danza }\end{array}$ & $\begin{array}{l}\text { Expresión } \\
\text { Musical }\end{array}$ & $\begin{array}{l}\text { Música/ } \\
\text { Plástica }\end{array}$ & $\begin{array}{l}\text { Música/ } \\
\text { Teatro }\end{array}$ & $\begin{array}{l}\text { Música/ } \\
\text { Danza }\end{array}$ \\
\hline UNAB & 0 & 0 & 1 & 0 & 0 & 0 & 1 & 0 \\
\hline UNACH & 1 & 0 & 0 & 0 & 5 & 0 & 0 & 0 \\
\hline PUCV & 0 & 0 & 0 & 1 & 0 & 0 & 0 & 1 \\
\hline UCSH & 1 & 0 & 0 & 0 & 1 & 0 & 0 & 0 \\
\hline UBO & 1 & 0 & 0 & 0 & 2 & 0 & 0 & 0 \\
\hline IP & 1 & 0 & 0 & 0 & 1 & 0 & 0 & 0 \\
\hline UMCE & 1 & 0 & 0 & 0 & 1 & 0 & 0 & 0 \\
\hline UDLA & 1 & 0 & 0 & 0 & 1 & 0 & 0 & 0 \\
\hline UPV & 1 & 0 & 0 & 0 & 1 & 0 & 0 & 0 \\
\hline UAH & 1 & 0 & 0 & 0 & 1 & 0 & 0 & 0 \\
\hline UC & 1 & 0 & 0 & 0 & 1 & 0 & 0 & 0 \\
\hline UDEC & 1 & 0 & 0 & 0 & 1 & 0 & 0 & 0 \\
\hline UDP & 1 & 0 & 0 & 0 & 1 & 0 & 0 & 0 \\
\hline UFT & 1 & 0 & 0 & 0 & 1 & 0 & 0 & 0 \\
\hline ULL & 0 & 0 & 0 & 1 & 0 & 0 & 0 & 1 \\
\hline $\mathrm{UCH}$ & 0 & 0 & 0 & 0 & 0 & 0 & 0 & 0 \\
\hline UMAY & 0 & 0 & 0 & 0 & 0 & 0 & 0 & 0 \\
\hline UNAP & 1 & 0 & 0 & 0 & 1 & 0 & 0 & 0 \\
\hline USS & 0 & 0 & 0 & 0 & 0 & 0 & 0 & 0 \\
\hline UTA & 1 & 0 & 0 & 0 & 1 & 0 & 0 & 0 \\
\hline UA & 0 & 0 & 0 & 0 & 0 & 0 & 0 & 0 \\
\hline USEK & 1 & 0 & 0 & 0 & 1 & 0 & 0 & 0 \\
\hline UCM & 1 & 0 & 0 & 0 & 1 & 0 & 0 & 0 \\
\hline
\end{tabular}

Fuente de Elaboración Propia

\section{Estrategia de Análisis de los Datos}

Para el análisis de los datos, se utilizó la estadística descriptiva con el apoyo del programa informático SPSS 25.0. Específicamente, se analizaron 23 mallas curriculares de educación parvularia de instituciones chilenas, correspondiente al $15 \%$, del total de 154 carreras ofertadas en el país (Falabella y Rojas, 2008), evidenciando el carácter representativo de la muestra. Además, se pudo identificar cuantas mallas curriculares contenían la asignatura de Expresión Musical, ya sea de forma autónoma, o bien en combinación con otra disciplina artística: música y plástica, música y teatro, música y danza. De igual manera, tras la revisión es posible señalar cuantas formaciones carecían de la asignatura Expresión Musical en sus mallas.

\section{RESULTADOS}

La revisión bibliográfica del instrumento BCEP 2018, revelatresaspectos relevantes paranuestro análisis. En primer lugar coarta la planificación de aprendizajes que combinen los tres ámbitos de experiencias de forma transversal (desarrollo personal y social, comunicación integral, interacción y comprensión del entorno). Además, se considera el núcleo de lenguaje artístico (danza, música, teatro, plástica) como un todo, mermando significativamente la profundización pedagógica y técnica en cada área artística. Además, no se encontró bibliografía actualizada 
sobre la educación musical y sus metodologías actuales (MINEDUC, 2018), lo que revela un desconocimiento y desactualización importante acerca del estado del arte sobre educación musical y neurociencias.

La revisión curricular nos muestra un bajo porcentaje de horas lectivas para la materia educación musical. Entre $1^{\circ}$ a $4^{\circ}$ año de educación básica sólo un $5,4 \%$, mientras que un
$2,6 \%$ para $5^{\circ}$ y $6^{\circ}$ año del mismo nivel en el total de horas lectivas para cada grado (MINEDUC, 2013).

El análisis de estos datos arrojó que del total de instituciones estudiadas, un $70 \%$ consideran la asignatura Expresión Musical de forma autónoma dentro de sus programas. Un 13\% de ellas imparte la asignatura en combinación con otras artes, mientras que un $17 \%$ no la considera dentro de sus planes (ver Figura 1).

\section{Porcentaje de Universidades que contienen la asignatura expresión musical}

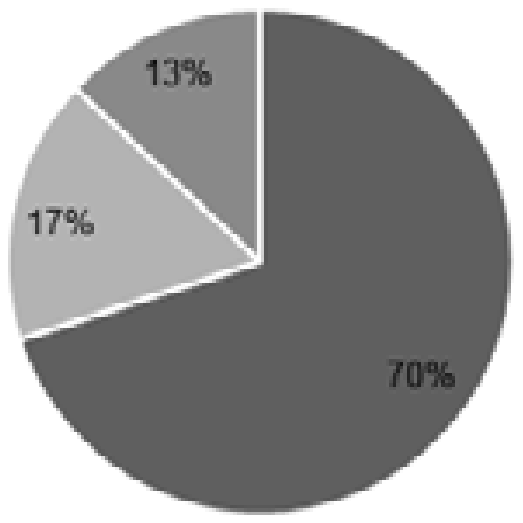

- Curricula con expresión musical

- Curricula con expresión musical combinada

- Curricula sin expresión musical

Figura 1. Porcentaje de universidades que contienen la asignatura expresión musical.

Esta revisión permite detectar que de las instituciones de educación superior analizadas, y que imparten la asignatura Recursos Musicales, un $92 \%$ de ellas sólo le asigna un semestre para su desarrollo (equivalentes a 2 créditos), mientras que solo dos universidades superan este margen, es decir, una con dos semestres (equivalentes a 4 créditos), y otra le dedica 5 semestres (equivalentes a 10 créditos) respectivamente. 


\section{$\mathbf{N}^{\circ}$ de Semestres que Universidades dedican a la Formación en Expresión Musical en Educación Parvularia}

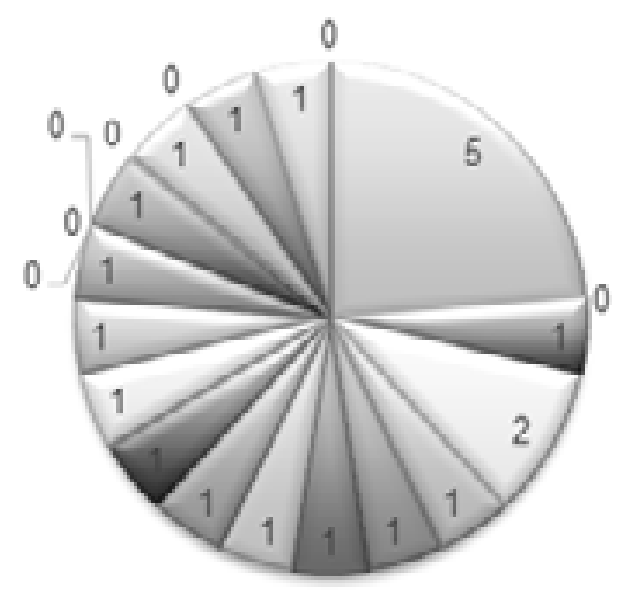

\begin{tabular}{|c|c|c|c|c|c|c|c|}
\hline UUNAB & $\triangle U N A C H$ & $\triangle P U C V$ & $\triangle \mathrm{UCSH}$ & UUBO & UIP & $\triangle U M C E$ & \\
\hline & $\triangle \mathrm{UAH}$ & $\square \cup C$ & $\triangle$ UDEC & UUDP & घUFT & घULL & $\triangle \mathrm{UCH}$ \\
\hline UMAY & - UNAP & UUSS & IUTA & $\square \mathrm{UA}$ & -USEK & U UCM & \\
\hline
\end{tabular}

Figura 2. Número de semestres de instituciones que contienen la asignatura expresión musical.

\section{DISCUSIÓN}

En Chile estamos atravesando un cambio de paradigma iniciado por el estallido social del 18 de Octubre, en donde la ciudadanía comienza a involucrarse activamente en un proceso de reforma constituyente. Esta situación representa "una grieta profunda en las formas del saber de la política conocidas hasta el momento, que tiene la potencialidad de abrirse a lo múltiple" (Ramírez, Yañez-Urbina, Salinas, 2020, p.25), como también representa la oportunidad de realizar cambios estructurales en el sistema educativo obsoleto que aún nos condiciona. Arredondo (2020, p. 6), analizando la Autoridad Pedagógica en la educación musical chilena visualiza:

"Estos cambios, que demandan una reestructuración sistémica, sin duda provocan grandes oportunidades de introspección, en cuanto al análisis de la propia posición como sujeto dentro de la balanza del país, como también instancias de evaluación de las diversas instituciones que forman parte del diario vivir colectivo donde la educación, por supuesto, no queda al margen."

Este contexto nos empuja a la adopción de una perspectiva crítica, no solo de nuestra posición como sujetos sociales, sino también de las instituciones encargadas de la formación inicial docente en Chile. Por consiguiente, cobra relevancia preguntarse: ¿Cómo posicionar en los cargos públicos, profesionales expert@s en el ámbito educativo, que comprendan la relevancia de fortalecer la educación musical en la educación parvularia, para reestructurar el sistema curricular hegemónico?, ¿Qué modelo de pedagogía musical es más efectivo para el trabajo integral con niños y niñas?,

¿Cómo instalar políticas públicas de capacitación en el área musical, desde un enfoque práctico y transferible?, ¿Qué beneficios podemos encontrar en una generación que tuvo una infancia musical significativa? ¿Por qué razón 
la educación musical quedó al remolque del sistema escolar chileno? ¿Cuánto interés existe en la comunidad científica para abordar y repensar críticamente estas problemáticas?

Las instituciones que forman a las profesionales de la educación parvularia ( $\mathrm{y}$ profesionales de la primera infancia) se encuentran con la posibilidad histórica de generar un cambio relevante en sus planes de estudio, equiparando la carga horaria de asignaturas tradicionalmente hegemónicas como lenguaje y matemáticas (Espinoza 2016, en Arredondo, 2020), otorgando a las artes musicales al menos la obligatoriedad de formación, como también el apoyo a la investigación en esta área.

Resulta pues necesario ser partícipes activos en el proceso de transformación social, como académicos e investigadores, aportando desde las ciencias fundamentos sólidos para la construcción de un nuevo paradigma del sistema educativo.

\section{CONCLUSIONES}

La revisión bibliográfica es extensa respecto a los beneficios de la actividad musical en el cerebro (Patel, 2003; Peretz y Coltheart, 2003; Bangerter y Heath, 2004; Zatorre, Chen y Penhune, 2007; Cánovas, Estévez y Sánchez-Santed, 2008; Forgeard et al, 2008; Albornoz, 2009; Schlaug et al, 2009; Milovanov, 2009, 2010 y 2011; Dege et al., 2011; Soria-Urios, Duque y Garcia-Moreno, 2011; Meher et al., 2013; Collins, 2013; Roden et al. 2013 y 2014; Zuk et al. 2015; Fernández y García, 2015; Custodio y Cano-Campos, 2017; Benítez, Díaz y Justel, 2017; Jaschke, Honing. y Scherder, 2018; Orozco y Gil, 2018; RománCaballero y Lupiañez, 2019), sin embargo faltan políticas públicas que permitan una mayor influencia en el curriculum formal de todo el sistema educativo (Collins, 2013).

El porcentaje de universidades que no imparte la formación de recursos musicales en la carrera de educación parvularia, aún cuando, los beneficios del entrenamiento musical a temprana edad son elocuentes, parecen evidenciar la rigidez propia de un paradigma que urge por cambiar. Acerca de la estimulación temprana, Sandoval (2018) enuncia que "las prácticas pedagógicas monótonas y rígidas, muchas veces ancladas en planes y programas absolutamente desconectados de los saberes y experiencias previas acumuladas en los ambientes de origen del alumnado", evidencian la lógica escolar centrada en los resultados. Sin embargo ésta desidia para con el saber musical devela algo más fundante, el desconocimiento acerca de la potencialidad del ejercicio musical en cualquiera de los niveles en que éste se ejerza. Es claro, el ejercicio musical genera un cerebro más despierto, y más allá que la asignatura musical representaba la algarabía del sistema escolar, ubicándola a la periferia del currículum escolar (Arredondo, 2020), ésta se orienta no solo al desarrollo de habilidades blandas, también su ejercicio compromete competencias y habilidades duras que perfectamente habilita la construcción de un sujeto que sea competente con el sistema socio económico, donde el estudio musical, para quien quiera comprender su complejidad y beneficios, puede potenciar ampliamente la productividad en el complemento que significa el desarrollo de un sujeto integral.

Las estrategias pedagógicas y metodológicas que se utilizan para la formación musical parecen ser de relevancia para nuestro análisis. No es lo mismo el desarrollo de la musicalidad de un ser humano desde una perspectiva con enfoque técnico, ligado a la tradición de conservatorio, que encararla desde un enfoque práctico, la cual promueve aspectos como el trabajo colaborativo, el desarrollo de la escucha, la improvisación libre, la consciencia corporal entre otras virtudes (Aróstegui e Ivanova 2012; Aróstegui, 2011). En este sentido, resulta fundamental no solo cuestionarnos la cantidad de horas de formación, sino también la calidad, 
los contenidos, y las metodologías de aquella formación.

Los resultados del análisis de formación musical en carreras de educación parvularia chilena, contrastan significativamente con la hipótesis propuesta en esta revisión, ya que solo un $70 \%$ de las instituciones que participaron del estudio ofrecen la asignatura Expresión Musical o su equivalente en autonomía de otras artes, y sólo un $7 \%$ del total ofrece dos o más semestres de formación musical en carrera.

A la música se puede llegar desde diferentes lugares, no obstante, es ineludible el hecho que la expresión musical requiere a lo menos un año de estudio para comenzar a manejar ciertos elementos con propiedad y de forma funcional en el trabajo con niños y niñas. Del análisis de formación musical a los planes de estudio de carreras chilenas de educación parvularia, se puede inferir que el $91 \%$ presentó un semestre de la asignatura de Expresión Musical, con o sin combinación con otras disciplinas, lo que resulta insuficiente para que el alumnado pueda desarrollar competencias reales que sean transferibles en estrategias efectivas de recursos musicales en el trabajo con párvulos.

Finalmente, el presente trabajo muestra una tendencia curricular imperante que tiende a cercenar signitivamente la formación musical en los procesos de escolarización chilenos, mermando con ello, las posibilidades de desarrollo y expresión musical que los educandos podrían alcanzar tempranamente a través de la expresión y la estimulación musical dentro y fuera de la institución escolar.

\section{REFERENCIAS BIBLIOGRÁFICAS}

Angel-Alvarado, R. y Lira-Cerda, J. P. (2017). Instalaciones y recursos educativos para la educación musical según la representación social de los estudiantes chilenos. Revista Electrónica de LEEME, (20), 19-31. doi:10.7203/ LEEME.40.10412

Arredondo C. (2020). Autoridad pedagógica en la educación musical chilena: desde la periferia curricular a la revisión del sistema en medio del estallido social. Átemus, 4(8), 5-13. Consultado de https://revistas.uchile.cl/index.php/atemus/ article/view/56025/59224

Aróstegui, J. L. (2016). Exploring the global decline of music education. Arts Education Policy Review, 117(2), 96-103. Doi: http://doi. org/10.1080/10632913.2015.

Aróstegui, J. L. (Ed.) (2014). La Música en Educación Primaria. Manual de formación del profesorado. Madrid: Dairea.

Aróstegui, J. L. (2011). Por un currículo contrahegemónico: de la educación musical a la música educativa* http://abemeducacaomusical. com.br/revista_abem/ed25/revista25_artigo2.p

Arostegui, J. y Ivanova, L. (2012). Didáctica de la expresión musical, una revisión del desarrollo del currículo. Barcelona: Red universitaria de investigación e innovación Educativa.

Albornoz, Y. (2009). Emoción, música y aprendizaje significativo. Educere, 13(44), 6773. Recuperado de https://dialnet.unirioja.es/ servlet/articulo?codigo $=3135378$

Banai, K. y Kraus, N. (2006). Neurobiology of (central) Auditory Processing Disorder and language-based learning disability. En G.D. Chermak y F. E. Musiek (Eds.), Handbook of Central Auditory Processing Disorder, vol. 1. Auditory Neuroscience and Diagnosis (pp. 89116). San Diego: Plural Publishing Inc. 
Bangerter, A. y Heath, C. (2004). The Mozart effect: tracking the evolution of a scientific legend. British Journal of Social. Psychology, 43(4), 605 - 623. Doi: 10.1348/0144666042565353.

Barraza, P. (2018). Música y educación: Derribando el mito de un saber prescindible. Sitio de noticias Universidad de Chile. Recuperado el 14 de octubre del 2019 en http://www. uchile.cl/noticias/149154/musica-y-educacionderribando-el-mito-de-un-saber-prescindible

Benítez, M., Díaz, V. y Justel, N. (2017). Beneficios del entrenamiento musical en el desarrollo infantil: una revisión sistemática. Revista internacional de educación musical, 5(1), 61-69. DOI: 10.12967/ RIEM-2017-5p061-069

Cánovas, D.A., Estévez, A.F. y SánchezSanted, F. (2008). El cerebro musical. Almería: Universidad de Almería.

Collins, A. (2013). Neuroscience meets music education: Exploring the implications of neural processing models on music education practice. International Journal of Music Education, 31(2), pp. 217-231. http://doi. org/10.1177/0255761413483081

Consejo de Rectores de las Universidades Chilenas (2019). Sistema de créditos académicos transferibles, 2019. Recuperado el 14 de octubre del 2019 de https://sct-chile.consejoderectores. cl/preguntas frecuentes.php\#uno

Custodio, N. y Cano-Campos, M. (2017). Efectos de la música sobre las funciones cognitivas. Revista de Neuro-psiquiatría, 80(1), 60-69.

Degé, F., Kubicek, C. y Schwarzer, G. (2011). Music lessons and intelligence: $A$ relation mediated by executive functions. Music Perception, 29(2), 195-201. https://doi. org/10.1525/mp.2011.29.2.195

Duarte, J.; Gargiulo, C. y Moreno, M. (2011)
Infraestructura escolar y aprendizajes en la Educación Básica Latinoamericana: Un Análisis a partir del Segundo Estudio Regional Comparativo y Explicativo de UNESCO. Washington: BID.

Elige Educar (2019). Educación parvularia en Chile: Estado del arte de la educación y desafíos. Una propuesta de Elige Educar. Santiago de Chile: Elige Educar. Recuperado de https:// eligeeducar.cl/wp-content/uploads/2019/09/ Elige-Educar.-Educacion-parvularia-en-Chile_ VF.pdf

Estévez, M. y Rojas, A. (2017). La Educación Artística en la Educación Inicial: Un Requerimiento de la formación del profesional. Revista Universidad y Sociedad, 9(4), 114-119.

Falabella, A. y Rojas, M. (2008). Algunas tendencias curriculares en la formación de educadores de párvulos. Calidad En La Educación, (29), 160-191. DOI: https://doi. org/10.31619/caledu.n29.192

Fernández, B. y García, J. (2015). De la Psicología de la Música a la Cognición Musical: Historia de una Disciplina ausente en los Conservatorios. ArtsEduca, 10, 38-61. Recuperado de https:// dialnet.unirioja.es/descarga/articulo/5443243. pdf

Forgeard, M.; Winner, E.; Norton, A.; Schlaug, G. y Fitch, T. (2008). Practicing a Musical Instrument in Childhood is Associated with Enhanced Verbal Ability and Nonverbal Reasoning. Plos one, 3(10), e3566. https://journals.plos.org/plosone/ article?id=10.1371/journal.pone. 0003566

Guerra, P.; Figueroa, I.; Salas, N.; Arévalo, R. y Morales, A. (2017). Desarrollo Profesional en Educadoras de Párvulos: Análisis de una Experiencia Formativa desde la Investigaciónacción y la Interacción Mediada. Estudios pedagógicos (Valdivia), 43(3), 175-192.

Hernández, R.; Fernández C. y Baptista, M. 
(2016). Metodología de la Investigación 6ta edición. Ciudad de México: McGraw-Hill.

Jaschke, A.; Honing, H. y Scherder, E. (2018). Longitudinal Analysis of Music Education on Executive Functions in Primary School Children. Frontiers in Neuroscience, 12 (103). https://doi. org/10.3389/fnins.2018.00103

Kerlinger, F. N. (2002). Investigación del comportamiento. Ciudad de México: McGrawHill.

Leonard, K.; Hafford-Letchfield, T. y Couchman, W. (2018). The impact of the arts in social work education: A systematic review. Qualitative Social Work, 17(2), 286-304. https://doi. org/10.1177/1473325016662905

Mehr, S. A.; Schachner, A.; Katz, R.C. y Spelke, E. S. (2013). Two Randomized Trials Provide No Consistent Evidence for Nonmusical Cognitive Benefits of Brief Preschool Music Enrichment. PLoS ONE 8(12), e82007. Doi:10.1371/journal. pone. 0082007

Milovanov, R. (2009). The Connectivity of Musical Aptitude and Foreign Language Learning Skills: Neural and Behavioural Evidence. University of Turku, Finland. Turku: University of Turku.

Milovanov, R.; Pietilä, P.; Tervaniemi, M. y Esquef, P. (2010). Foreign language pronunciation skills and musical aptitude: $A$ study of Finnish adults with higher education. Learning and Individual Differences. Learning and Individual Differences, 20(1), 56-60. https:// doi.org/10.1016/j.lindif.2009.11.003

Milovanov, R. y Tervaniemi, M. (2011). The interplay between musical and linguistic aptitudes: a review. Frontiers in Phsicology, 2(321), 1-6. Doi: 10.3389/fpsyg.2011.00321

Ministerio de Educación (2013). Bases curriculares: Educación básica. Santiago de Chile: Unidad de Currículum y Evaluación.
Santiago de Chile: MINEDUC.

Ministerio de Educación (2018). Bases Curriculares de Educación Parvularia. Santiago de Chile: Subsecretaría de Educación Parvularia, MINEDUC.

Orozco G. y Gil K. (2018) Beneficios cognitivos cerebrales de la práctica de la danza Ciencia \& Futuro, 8(3), 142-160.

Patel, A. (2003). Language, Music, Syntax and the Brain. Nature Neuroscience, 6(7), 674-681.

Peñalba, A. (2017). La defensa de la educación musical desde las neurociencias. Revista Electrónica Complutense de Investigación en Educación Musical, (14), 109-127 http://dx.doi. org/10.5209/RECIEM.54814

Peretz, I. y Coltheart, M. (2003). Modularity of Music Processing. Nature Neuroscience, 6(7), 688-690.

Piscoya, L. (2009a). El Proceso de la Investigación Científica. Lima: UIGV.

Popper, K. (2008). La Lógica de la Investigación Científica. Madrid: Tecnos.

Ramírez, C.; Yañez-Urbina, C. y Salinas, I. (2020). Chile, la Democracia se Acabó: Crisis Institucional en el 18-O chileno. Reresentaciones. Investigación en Comunicación, (12), 6-28.

Roden, I.; Grube, D.; Bongard, S. y Kreutz, G. (2014). Does music training enhance working memory performance? Findings from a quasiexperimental longitudinal study. Psychology of Music, 42(2), 284-298. https://doi. org/10.1177/0305735612471239

Roden, I.; Könen, T.; Bonnard, S.; Frankenberg, E.; Friedrich, E. y Kreutz, G. (2014). Effects of Music Training on Attention, Processing 
Speed and Cognitive Music Abilities_Findings from a Longitudinal Study. Applied Cognitive Psychology, 28(4), 545-557. https://doi. org/10.1002/acp.3034

Román-Caballero, R. y Lupiáñez, J. (2019). El impacto cognitivo de la práctica musical: Explorando las ventajas de un mundo musicalmente activo. Ciencia Cognitiva, 13(1), 21-23.

Sandoval, E. (2018). La Estimulación Temprana en la Infancia: Una Tarea Relevante y Necesaria para el Desarrollo Integral. Otras Voces en Educación. Recuperado de http:// otrasvoceseneducacion.org/archivos/290431

Soria-Urios, G.; Duque, P. y García-Moreno, J. (2011a) Música y Cerebro: Fundamentos Neurocientíficos y Trastornos Musicales. Revista de Neurología, 52(1), 45-55. DOI: https://doi. org/10.33588/rn.5201.2010578

Soria-Urios, G.; Duque, P. y García-Moreno. J. (2011 b). Música y cerebro (II): evidencias cerebrales del entrenamiento musical. Revista de Neurología, 53(12), 739-746. DOI: https://doi. org/10.33588/rn.5312.2011475

Zatorre, R.; Chen, J. y Penhune, V. (2007). When the brain plays music: auditory-motor interactions in music perception and production. Nature Reviews Neuroscience, 8, 547-558. https://doi.org/10.1038/nrn2152

Zuk, J.; Benjamin, C.; Kenyon, A. y Gaab, N. (2014). Behavioral and Neural Correlates of Executive Functioning in Musicians and NonMusicians. PLoS ONE 9(6), e99868. https://doi. org/10.1371/journal.pone.0099868 